\title{
Single-cell atlas maps cell-specific gene changes in Alzheimer disease
}

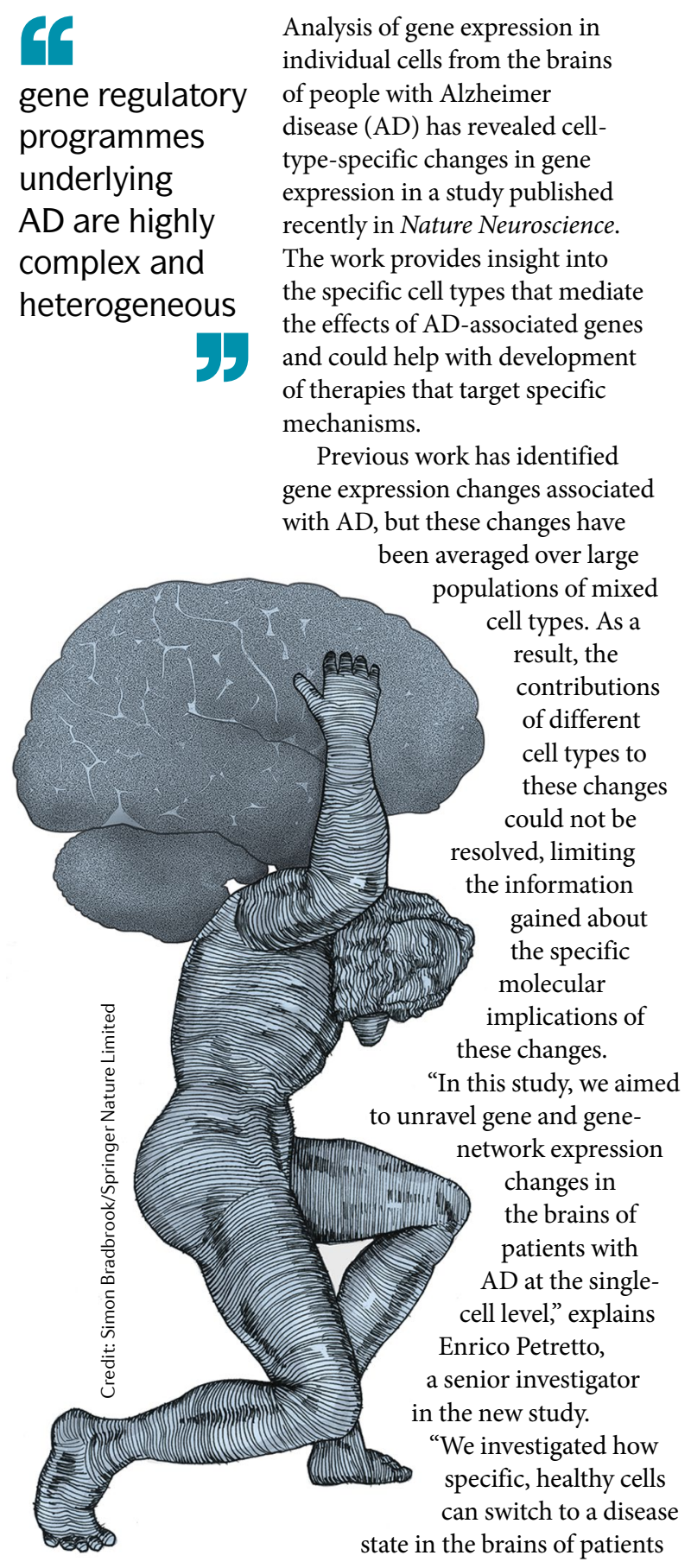

with $\mathrm{AD}$ and what the mechanistic underpinnings of these 'cell state transitions' in disease are."

Petretto and colleagues used RNA sequencing to analyse gene expression in individual cells in the entorhinal cortex of six patients with $\mathrm{AD}$ and six controls. In total, the transcriptomes of 13,214 nuclei were analysed. Reliable genetic markers were identified to classify the cells as microglia, astrocytes, neurons, oligodendrocyte progenitor cells, oligodendrocytes or endothelial cells. Gene expression patterns in cells from patients with $\mathrm{AD}$ were then compared with those in cells from controls.

The analysis identified nine clusters of gene expression patterns that differed between patients with $\mathrm{AD}$ and controls. Some of these changes were specific to certain cell types, particularly astrocytes, endothelial cells and microglia, and others were seen in multiple cell types. Furthermore, analysis of each cell type revealed multiple subclusters for each, and cells from brains with or without $\mathrm{AD}$ mostly segregated into different clusters.

The researchers also combined their data with data from previous genome-wide association studies to investigate the cell specificity of changes in $\mathrm{AD}$-associated gene expression. Changes in expression of many of these genes were predominantly seen in one cell type, whereas others were co-ordinated between multiple subclusters. For some genes, expression changes differed between cell types. For example, in tissue from patients with $\mathrm{AD}, A P O E$ was downregulated in subclusters of oligodendrocyte progenitor cells, oligodendrocytes and astrocytes, but upregulated in a microglial subcluster.

"Our study reveals that gene regulatory programmes underlying $\mathrm{AD}$ are highly complex and heterogeneous, demonstrating cell subtype-specific regulation and cell identity changes," explains Petretto. This insight, he continues, provides new information that could help with developing targeted therapies.

"In the past, when we have only been able to track changes over the total population of cells, it has been impossible to see how each cell type has contributed to the changes that result in $\mathrm{AD}$ so treatments have not been developed to target these cell type-specific processes," he explains. "Our research, along with that of others in the field, has allowed us to see which gene regulatory and functional processes in which cell types we need to target treatments to in order to get the best benefit."

The team has created the Single-cell Atlas of the Entorhinal Cortex in Human Alzheimer's Disease online to provide access to their data, which can be explored to see cell-specific gene expression, cell identities and markers. They hope that this atlas will facilitate further discoveries that reveal the functional consequences of genetic changes.

Ian Fyfe

ORIGINAL ARTICLE Grubman, A. et al. A singlecell atlas of entorhinal cortex from individuals with Alzheimer's disease reveals cell-type-specific gene expression regulation. Nat. Neurosci. 22, 2087-2097 (2019)

RELATED LINKS

Single-cell atlas of the Entorhinal Cortex in Human Alzheimer's Disease: http://adsn.ddnetbio.com 